\title{
Neues von den KVG-Kommissionen des Bundesamtes für Gesundheit (BAG)
}

Maya Züllig

Korrespondenz:

Sektion Medizinische Leistungen BAG

Kranken- und Unfallversicherung Abt. Leistungen Schwarzenburgstrasse 165 CH-3097 Liebefeld

sml@bag.admin.ch
Der Bundesrat hat im Rahmen des Projektes Bundesverwaltungsreform das Kommissionswesen gestrafft und dabei rund einen Viertel der 200 ausserparlamentarischen Kommissionen aufgehoben. Im Bereich der Krankenversicherung wurden einerseits die Eidgenössische Kommission für Grundsatzfragen der Krankenversicherung (EGK) und die Eidgenössische Kommission für allgemeine Leistungen (ELK) sowie andererseits die Eidgenössische Analysenkommission (ALK) und die Eidgenössische Kommission für Mittel und Gegenstände (MiGeLK) aufgehoben und zu zwei neuen Kommissionen zusammengelegt: zur Eidgenössischen Kommission für allgemeine Leistungen und Grundsatzfragen (ELGK) und zur Eidgenössischen Kommission für Analysen, Mittel und Gegenstände (EAMGK). Letztere setzt zwei Ausschüsse ein: einen für Analysen (EAMGK-AL) und einen für Mittel und Gegenstände (EAMGKMiGeL). Mit der Zusammenlegung der Kommissionen sollen Synergien besser genutzt und die Arbeit effizienter gestaltet werden.

Der Bundesrat hat in der Verordnung über die Krankenversicherung (KVV) die maximale Grösse der neuen Kommissionen vorgegeben und festgelegt, dass die ELGK aus 20 Mitgliedern und die EAMGK aus 19 Mitgliedern bestehen soll. Dies bedeutet, dass bei der Besetzung der Kommissionssitze aus dem Kreis der bisherigen Vertretungen eine Auswahl getroffen werden musste, wobei der Wahrung der Repräsentativität und einer möglichst breiten Abstützung der Kommission grosses Gewicht zukam (www.admin.ch $\rightarrow$ Dokumentation $\rightarrow$ Gesetzgebung $\rightarrow$ Systematische Sammlung $\rightarrow$ 832-102 $\rightarrow$ Artikel 37a-g). Sofern die besonderen Anliegen einer Vertretung nicht von seiten der gewählten Vertretungen in die Kommissionen oder Ausschüsse eingebracht werden können, besteht die Option, die Anliegen in den für sie relevanten Fragestellungen als Experte einzubringen.

Die Fachkommissionen beraten das Eidgenössische Departement des Innern (EDI) bei der Bezeichnung eines einheitlichen, für alle Versicherer verbindlichen Leistungskatalogs nach Vorgabe des Bundesgesetzes über die Krankenversicherung (KVG). Dieser wird mit Ausnahme der Arzneimittel in der departementalen Kranken- pflegeleistungsverordnung (KLV) und ihren Anhängen festgelegt. Als massgebende Kriterien für eine Aufnahme nennen das KVG und die dazugehörigen Verordnungen die Wirksamkeit, Zweckmässigkeit und Wirtschaftlichkeit.

Die laufende Anpassung des Leistungskatalogs erfordert einen transparenten und standardisierten Beurteilungs- und Entscheidungsprozess. Dieser ist über Jahre entwickelt und laufend verfeinert worden. Das seinerzeit zuständige Bundesamt für Sozialversicherungen (BSV) hat bereits Ende der 90er Jahre international beachtete Pionierarbeit geleistet und die Grundsätze in einem Handbuch für Antragstellende festgehalten. Nach längeren Vorarbeiten hat die für die wissenschaftlichen und administrativen Kommissionssekretariate zuständige Facheinheit des BAG, die Sektion Medizinische Leistungen, die Vorgaben für Antragstellende neu aufgelegt: Das Handbuch wurde vollständig zu Modulen in Formularform umgearbeitet und leitet neu durch einen zweistufigen Antragsprozess (www.bag. admin.ch $\rightarrow$ Themen $\rightarrow$ Krankenversicherung $\rightarrow$ Leistungen und Tarife $\rightarrow$ Ärztliche Leistungen).

\section{Informationsveranstaltung}

Die Neuerungen im Antragsverfahren für die Vergütung von neuen oder umstrittenen medizinischen Leistungen, Laboranalysen sowie Mitteln und Gegenständen werden am Freitag, 13. Juni 2008 , von 9.30 bis $12.30 \mathrm{Uhr}$ im Inselspital in Bern vorgestellt.

Zielpublikum: ärztliche und labormedizinische Fachgesellschaften, Spitäler, Apotheken, Hersteller und Vertriebsfirmen, Beratungsunternehmen, Versicherer sowie Behörden und Verbände.

Die Teilnahme ist kostenlos.

Die Vorträge auf Deutsch werden simultan auf Französisch übersetzt.

Detailprogramm und Anmeldeformular: siehe www.bag.admin.ch $\rightarrow$ Aktuell $\rightarrow$ Veranstaltungen.

Anmeldefrist: 31. Mai 2008 